Meta

Journal des traducteurs

Translators' Journal

\title{
Le signifié et le concept dans la dénomination
}

\section{Marcel Diki-Kidiri}

Volume 44, numéro 4, décembre 1999

URI : https://id.erudit.org/iderudit/002566ar

DOI : https://doi.org/10.7202/002566ar

Aller au sommaire du numéro

\section{Éditeur(s)}

Les Presses de l'Université de Montréal

ISSN

0026-0452 (imprimé)

1492-1421 (numérique)

Découvrir la revue

Citer cet article

Diki-Kidiri, M. (1999). Le signifié et le concept dans la dénomination. Meta, 44(4), 573-581. https://doi.org/10.7202/002566ar

\section{Résumé de l'article}

Le signe linguistique est arbitraire. Sans chercher expressément à remettre en cause la célèbre assertion saussurienne qui concerne essentiellement sinon uniquement le rapport entre le signifiant et le signifié, nous nous intéresserons à la différence que l'on est fondé à établir entre ce dernier et ce qu'est un concept.En nous appuyant sur des données tirées de plusieurs langues et cultures tant africaines qu'européennes, nous montrerons que bien que les notions de "concept" et de "signifié" puissent toutes les deux évoquer les mêmes produits culturels de l'esprit humain, le concept semble renvoyer à plus d'objectivité et donc à plus d'universalité dans la représentation des choses, tandis que le signifié, lui, paraît plus étroitement dépendant des perceptions particulières à chaque culture. 


\title{
Le signifié et le concept dans la dénomination'
}

\author{
MARCEL DIKI-KIDIRI \\ CNRS (LLACAN), Meudon, France
}

\section{RÉSUMÉ}

Le signe linguistique est arbitraire. Sans chercher expressément à remettre en cause la célèbre assertion saussurienne qui concerne essentiellement sinon uniquement le rapport entre le signifiant et le signifié, nous nous intéresserons à la différence que l'on est fondé à établir entre ce dernier et ce qu'est un concept.

En nous appuyant sur des données tirées de plusieurs langues et cultures tant africaines qu'européennes, nous montrerons que bien que les notions de «concept» et de «signifié» puissent toutes les deux évoquer les mêmes produits culturels de l'esprit humain, le concept semble renvoyer à plus d'objectivité et donc à plus d'universalité dans la représentation des choses, tandis que le signifié, lui, paraît plus étroitement dépendant des perceptions particulières à chaque culture.

\begin{abstract}
Without challenging the Saussurean assertion as to the arbitrariness of the sign, based primarily if not solely on the relationship between "signifier" and "signified", it is worthwhile to establish the difference between signifier and concept.

Using data from several languages and cultural contexts, European as well as African, this article aims to demonstrate that although concept and signified both conjure up identical cultural realities, the notion of concept seems to be more objective, and therefore more universal in terms of representing things, whereas that of signified seems to be closely linked to cultural perception. The signified may change from one culture to another; it may also change over time within the same culture. For a given object, the concept may be assumed to be the essential idea, the principle or rather the archetype, and the signified the "vantage" point — one point of view among many.
\end{abstract}

\section{INTRODUCTION}

En travaillant sur l'instrumentalisation terminologique des langues africaines pour l'expression de réalités modernes qui n'existaient pas ou n'étaient pas prééminentes dans le monde des cultures traditionnelles africaines, nous sommes quotidiennement confrontés à la complexité de l'unité terminologique, à savoir le terme. Un certain nombre de contraintes s'imposait d'emblée à nous qui cherchions à dénommer des réalités nouvelles dans nos langues:

- Nous sommes ici dans un cas de figure où les réalités nouvelles à dénommer existent indépendamment de notre culture, étant le plus souvent créées en Occident. Nous nous sommes interrogé, d'une part, sur la nature de ces réalités nouvelles et, d'autre part, sur comment elles ont été dénommées dans les cultures occidentales où elles ont été créées. Ces dénominations, réputées précises et scientifiques lorsqu'il s'agit de produits technologiques ou de domaines de spécialité, le sont-elles parce qu'elles désignent de façon précise l'essence des choses telle qu'elle nous est révélée par la science en tant que connaissance objective? Si tel n'était pas le cas, qu'est-ce qui fait que ces dénominations «fonctionnent»? Nous espérons par cette démarche mieux comprendre comment nous pourrions dénommer au mieux les réalités nouvelles dans nos langues respectives. 
- Un deuxième cas de figure est celui de réalités anciennes en Afrique, mais qui n’avaient pas jusque-là fait l'objet d'études avancées dans les cultures africaines traditionnelles. Au contact de l'Occident, la connaissance de certaines de ces réalités est devenue importante pour le développement économique, par exemple, les insectes ravageurs de coton. Ici, nous procédons à trois types d'observation: la nature intrinsèque de ces réalités, les dénominations occidentales et les dénominations traditionnelles africaines. Par ces observations, nous cherchons à savoir dans quelle mesure les dénominations occidentales sont motivées par la réalité en soi ou non, et donc dans quelle mesure les dénominations africaines existantes sont encore opérationnelles, donc récupérables. L'évaluation de celles-ci permet d'orienter l'activité de mise à niveau terminologique. Par-delà ces cas d'application, la question fondamentale est donc bien de savoir qu'est-ce que dénommer veut dire, et qu'est-ce qu'une «bonne» dénomination. On espère que sachant cela, on saura mieux comment aménager une «bonne» terminologie.

- Dans tous les cas de figure, les termes produits, qu'ils soient néologiques ou non, sont des unités linguistiques censées faire partie intégrante d'une langue naturelle et destinées à servir à la communication aussi bien dans le cadre d'activités professionnelles (ex. culture du coton) que dans celui du transfert des connaissances (ex. scolarisation, alphabétisation, formation). Le terme est donc un signe linguistique qui permet, dans une langue naturelle, l'expression et la communication d'une connaissance spécialisée.

Si donc le terme est bien un «signe linguistique» à la saussurienne, force est de reconnaître que la seule relation binaire signifiant/signifié est insuffisante pour décrire le terme dans toute sa complexité. Dans une communication récente, Teresa Cabré (1997) suggère une description tridimensionnelle du terme: une linguistique, une sociale et une utilitaire. En ne restant que sur la dimension linguistique, nous pensons qu'il y a encore lieu de distinguer trois axes de description qui s'articulent autour du signifiant, du signifié et du concept.

Sur l'axe du signifiant, on traitera de toutes les questions relatives à la forme (ou aux différentes formes) du terme, à savoir la formation des mots, les formants, les radicaux, l'homonymie, la synonymie, etc. Lorsqu'on est amené à créer de la néologie terminologique, ces questions de formes ne sont évidemment pas à négliger si l'on veut produire des termes «bien formés» ayant toutes les chances de s'implanter assez aisément. Bien souvent, une bonne formation du signifiant ne suffit pas à faciliter l'implantation du néologisme si les autres aspects ne sont pas optimisés.

C'est au niveau du signifié que se situe la problématique de la construction du sens, ce qui implique, très souvent, une « reconceptualisation» de l'objet à dénommer en fonction des perceptions culturelles. C'est aussi à ce niveau du signifié que se situe au mieux la question de la polysémie en relation avec la construction du sens. Il arrive en effet couramment que les méthodes de la construction du sens (en particulier la métaphore et la métonymie) entraînent une polysémie dont il est nécessaire de rendre compte ici.

Enfin, c'est au niveau du concept que l'on se pose l'essentiel des questions relatives à la cognition et à la nature du savoir, de son objectivité et de son universalité vraies ou vraisemblables. De nombreuses recherches cognitivistes démontrent amplement le caractère toujours construit des concepts, y compris ceux qui nous paraissent les plus objectifs, c'est-à-dire les plus «indépendants de toute subjectivité ». C'est en explorant avec les cogniticiens la nature des relations entre un concept et divers objets concrets ou abstraits qu'il est censé représenter que l'on peut, semble-t-il, établir la portée de la dénomination. Et pour finir, lorsqu'on est amené à faire une distinction 
entre les mots ordinaires de la langue dite générale et les termes propres à une spécialité, un domaine avancé du savoir, il y a lieu de prendre ensemble cette triple articulation du terme autour du signifiant, du signifié et du concept.

\title{
1. CONCEPT ET CLASSE D'OBJETS
}

Si nous considérons un objet fabriqué, par exemple une maison, un ordinateur, une bicyclette ou un pot, on dira que cet objet a été conçu avant d'être réalisé. Cette conception peut se traduire en une série de réflexions, d'études, de calculs, etc., aboutissant à une représentation mentale idéale de ce qu'est censé être l'objet du point de vue de son inventeur. C'est cette représentation mentale que nous appellerons ici le «concept» de l'objet parce qu'il en exprime l'idée essentielle, le principe, l'archétype ${ }^{2}$. C'est un tel concept qui est protégé par la loi au nom de la propriété intellectuelle lorsqu'un inventeur dépose un brevet. Ce concept peut être réduit sous une forme écrite qu'on appelle "plan de réalisation». Chaque objet réalisé grâce à ce plan est une instance concrétisée du concept. Outre que tous ces objets partagent les attributs essentiels du concept, chacun d'eux peut avoir des attributs propres dits "personnalisés» ou "localisés», qui peuvent justifier une structuration fractale en classes d'objets. Cette variation commence d'ailleurs souvent dès le niveau de la conceptualisation en donnant une famille de concepts. Pensez à la production d'une gamme de voitures qui prévoit dès la conception plusieurs modèles dans la même gamme et plusieurs variantes dans le même modèle.

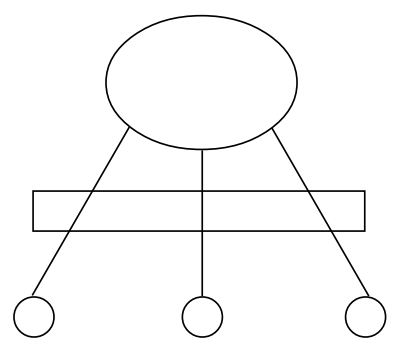

\author{
Concept (archétypique) \\ Images symboliques (schémas, plans) \\ Classes d'objets
}

Parce que ce type de concept est à la base de la création (production, construction, existence) des objets ainsi conçus, nous sommes tentés de le considérer comme l'expression de ce qu'ils sont intrinsèquement, indépendamment de toutes contingences. Il est sans doute plus prudent et probablement plus exact de considérer les concepts donnant lieu à des inventions technologiques comme représentant les archétypes de ces inventions. L'archétype occupe cette position privilégiée d'être le « concept premier» qui contient toute l'idée essentielle d'un objet imaginé et fabriqué par l'homme. L'archétype exprime en quelque sorte la vision de l'inventeur, une vision qui ne se concrétise que par la fabrication des objets ainsi conçus. Dans la suite de cet exposé, nous utiliserons l'expression concept archétypique pour parler de ce type de concept qui correspond à une représentation mentale structurante de classes d'objets et traduisibles en images symboliques (schémas ou plans).

Lorsque, pour de multiples raisons (usage, évolution, distance culturelle, etc.), les 
produits technologiques sont perçus ou présentés autrement que dans la vision de leur inventeur (c'est-à-dire leur concept archétypique), on dira qu'ils ont subi une reconceptualisation en fonction des nouvelles perceptions, car, dans certains cas, celles-ci peuvent entraîner une véritable recatégorisation des classes d'objets.

Lorsqu'il s'agit de caractériser des objets naturels non créés par l'homme: animaux, végétaux, minéraux, esprits, astres, «forces de la nature», etc., la question centrale est la catégorisation, comme le montrent très bien toutes les recherches sur la cognition, la prototypicalité, etc. Typiquement, lorsqu'on trouve un animal inhabituel, le degré de connaissance encyclopédique que l'on peut rassembler sur lui permet de le catégoriser avec d'autant plus de précision que ce degré est élevé. Ainsi, la baleine avait d'abord été classée comme un poisson avant de rejoindre la catégorie des mammifères. De même, les amphisbènes ont été classés avec les serpents, puis avec les vers de terre avant de se voir attribuer une catégorie spécifique pour eux tout seuls. La biologie qui, comme toutes les sciences, est parcourue par plusieurs courants de pensée, ne nous apporte pas la garantie absolue qu'il existe, dans la nature, des classes d'objets naturelles strictement objectives, indépendamment donc de toute perception humaine. Puisque l'homme n'a pas créé la baleine, le concept qu'il en aura ne peut être que construit, de façon déductive, à la hauteur de la somme de connaissances qu'il peut accumuler sur elle en l'observant. Il se crée ainsi une image mentale schématique de la baleine comparable à celle que se crée l'inventeur d'un produit. Cette image mentale est censée intégrer suffisamment de traits typiques de la baleine pour qu'on la reconnaisse quand on en rencontre une. Le concept de «baleine» que sous-tend cette reconstruction symbolique qu'est l'image mentale de la baleine n'est en rien différent d'un concept archétypique, en dépit de l'inversion du parcours (objets vers concept ici, concept vers objets dans le cas des artefacts).

Que l'on chemine du concept vers les objets (artefacts) ou des objets (espèces naturelles) vers le concept, on a toujours les trois composantes:

- concept archétypique (archétype, pool de traits distinctifs, structures);

- schémas symboliques (plans de réalisation, images mentales);

- classes d'objets (catégorisation à des fins d'identification et de connaissance).

La question cruciale qui se pose ici est de savoir si les catégories que nous établissons dans notre esprit, que ce soit à la suite de nos observations expérimentales ou par l'activité propre de notre esprit (éclair de génie de l'inventeur), nous donnent vraiment accès à la connaissance des choses telles qu'elles sont dans le monde extérieur à notre esprit. On en vient finalement à chercher à en savoir plus sur le fonctionnement même de l'esprit humain, notamment comment il s'y prend pour «connaître». De nombreuses théories de la connaissance ont été élaborées par les cogniticiens et les sémanticiens, chacune apportant sa pierre à la science du savoir. $\mathrm{Si}$ cette étude intéresse le terminologue, c'est en autant qu'elle lui permet de décrire, par exemple dans une définition en terminographie, les composantes essentielles (ou prototypiques) d'un objet de connaissance spécialisée, qu'il s'agisse d'un artefact ou d'une espèce naturelle. Il s'agit ici d'une problématique de caractérisation et de catégorisation. Mais la question de la dénomination proprement dite sera traitée ailleurs, dans l'interaction entre le signifiant et le(s) signifié(s) organisé(s) par les perceptions culturelles. 


\section{DÉNOMINATION ET PERCEPTIONS CULTURELLES}

Il est courant qu'un produit porte un nom de code au cours de sa conception et un autre nom, «commercial» cette fois, lors de sa mise sur le marché, sans compter celui ou ceux que les utilisateurs (consommateurs) lui donneront. L'affaire devient encore plus complexe lorsque la terminologie est envisagée sous un angle multilingue! Nous commencerons donc par présenter des cas simples afin de bien mettre en lumière les phénomènes que nous voulons décrire.

\subsection{Polysémie et organisation prototypique du sens}

Le mot «souris» en français désigne prioritairement (ou prototypiquement ${ }^{3}$ ) un «quadrupède de la famille des rongeurs, appartenant au genre rat» (Littré 1994). Mais on lit dans le même article plusieurs autres sens figurés, dérivés, métaphoriques, etc. tels que:

- un homme qui a très peur ou qui éprouve un grand embarras;

- une couleur gris-argenté;

- un muscle charnu qui tient par un bout à la manche du gigot;

- l'espace qui est dans la main entre le pouce et l'index.

Il est à noter que ces deux derniers exemples relèvent du même domaine, l'anatomie. En informatique, comme on le sait, le mot désigne un dispositif électromécanique ou électro-optique de pointage et de saisie. Nous avons donc là six sens différents pour le même mot souris. C'est donc bien un cas de polysémie. La polysémie en tant que phénomène linguistique est suffisamment bien étudiée pour qu'on ne s'y attarde ici pour elle-même. Nous nous contenterons donc de souligner quelques points pertinents pour la dénomination.

Considérant que le signifiant «souris» (que nous symbolisons par S) a six signifiés dont un central $\left(S_{0}=\right.$ «rongeur sp.») et cinq non centraux $\left(S_{1}=\right.$ «homme peureux sp.», $S_{2}=$ «gris sp.», $S_{3}=$ «muscle sp.», $S_{4}=$ «espace sp.», $S_{5}=$ «pointeur sp.»), il nous faut préciser tout de suite que les indices, de 0 à 5 , que nous attribuons symboliquement à ces six sens, n'ont d'autre but que de les distinguer commodément, et n'impliquent aucune structure hiérarchique. On pourrait ordonner ces six sens en fonction de leur datation historique, ou de leur déduction logique s'il y en a une, mais cela n'apporterait pas grand-chose à notre propos. Il est facile, en effet, d'expliquer ces différents sens du mot «souris », notamment par l'analogie, la métonymie, la métaphore, etc. (cf. Lakoff et Johnson 1985). Mais ce qui nous importe ici, c'est de montrer en quoi la question de la dénomination est pertinente et distincte de celle de la catégorisation.

Du point de vue de la catégorisation, seul le petit animal désigné par le mot «souris» peut être classé comme un « rongeur». Les autres signifiés ne le peuvent pas. Ils renvoient à des catégories conceptuelles autres que celle de «rongeur». Un homme, même apeuré, n'est pas un rongeur, pas plus qu'un muscle mobile, etc. Par contre, l'homme apeuré ou embarrassé a un comportement qui, dans la culture française (ou francophone), est perçu comme similaire au comportement du rongeur. L'espace réduit entre le pouce et l'index a été perçu, toujours dans la même culture, comme évoquant un trou de souris et a été donc appelé ainsi. La couleur gris-argenté étant dominante chez les souris, la synecdoque se comprend. Pour le 
muscle comme pour le dispositif électronique, c'est la ressemblance de forme qui a suffi à permettre le rapprochement. Tous les signifiés dérivés n’ont aucun trait commun entre eux mais partagent chacun au moins un trait commun, plus ou moins symbolique, avec le signifié central (prototypique) qui renvoie au concept archétypique de «quadrupède rongeur du genre rat» désigné par le signifiant souris. La propagation des traits à partir du signifié central vers les autres signifiés est de type radial:
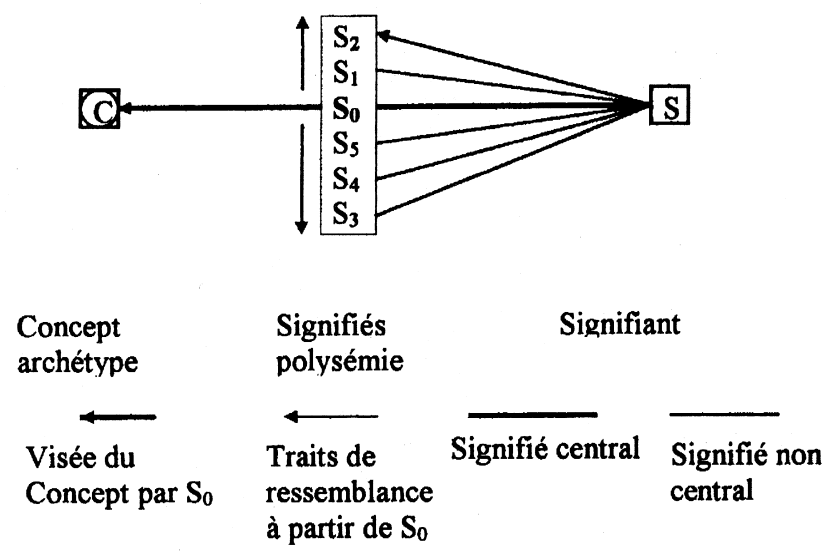

La position centrale (prototypique) du signifié $\left(\mathrm{S}_{0}\right)$ réflexe du concept archétypique $\left(\mathrm{C}_{0}\right)$ «quadrupède rongeur...», que désigne le signifiant $(\mathrm{S})$ «souris», peut varier avec le temps. Pour une génération d'enfants vivant dans une grande ville occidentale comme Paris et qui ont grandi avec les jeux vidéo, le mot «souris» évoque prioritairement (donc basiquement, prototypiquement) l'accessoire informatique. Et cette acception (signifié $\mathrm{S}_{5}$ ) supplante et relègue au second plan l'acception première d'animal «quadrupède rongeur». C'est dire que, même dans une polysémie, la centralité (ou la prototypicalité) d'une acception, d'un signifié, est directement dépendante du milieu social auquel appartient la majorité des personnes interrogées. Et si l'on considère l'évolution du sens d'un mot dans le temps, il n'est pas rare de constater qu'une acception nouvelle a complètement supplanté une autre plus ancienne totalement oubliée, sauf peut-être dans les encyclopédies. Ainsi le sens central du mot «clavier» aujourd'hui n'est plus celui de «porte-clés » et «clavicule» ne signifie plus "petite clé». Ce qui était une métaphore, et donc un signifié non central, est devenu avec le temps le sens premier, voire l'unique sens. Les acceptions oubliées ne sont, du reste, qu'éclipsées, comme mises en retrait. Il suffit de replacer un récit dans un contexte historique suffisamment ancien pour que ces acceptions refassent surface, pour éviter l'anachronisme. Les acceptions oubliées peuvent toujours être réactivées délibérément, même sans avoir besoin d'un contexte archaïsant. C'est ce que l'on fait chaque fois qu'on éprouve le besoin de préciser que tel mot est employé dans son sens étymologique.

Ainsi, dans une même langue, les signifiés qui se rattachent de façon polysémique à un même signifiant peuvent être organisés de manière prototypique aussi bien dans l'espace que dans le temps et en fonction de milieux sociaux relativement homogènes. Il s'ensuit qu'une acception non centrale dans un milieu donné, en un temps donné et en un endroit donné, peut occuper une position centrale dans un autre milieu, un autre temps ou un autre lieu. 


\subsection{Concepts et signifiés ou percepts}

La distinction entre concept, entendu comme structure cognitive de catégorisation, et signifié, envisagé comme le lieu des perceptions culturelles, s'impose encore plus nettement lorsqu'on compare la dénomination d'un même artefact dans plusieurs langues.

La bicyclette est appelée:

- gbâzâbängâ, «roues de caoutchouc», en sängö (Centrafrique);

- nàgàsót, "cheval de fer", en bambara (Mali);

- magu-mâkwanganya ${ }^{5}$, "quatre pieds », en lilikô (langue bantu de la zone D en République Démocratique du Congo).

Ces différentes appellations témoignent de la diversité dans la perception de l'objet bicyclette par des communautés de langues et de cultures différentes, ayant des passés différents. Les Centrafricains connaissaient la roue et avaient été, par ailleurs, soumis aux travaux forcés de la récolte du caoutchouc végétal dès les premières années de la colonisation de leur pays (vers 1910). Les roues de caoutchouc (pneu) de la bicyclette ont donc retenu particulièrement leur attention et motivé leur choix dans la dénomination de ce véhicule. De leur côté, les Bambara, qui connaissaient le cheval, ont perçu une ressemblance fonctionnelle entre cet animal et la bicyclette: les deux se montent à califourchon et permettent à l'homme de se déplacer plus rapidement qu'à pied. Par contre, seul ce dernier trait (déplacement plus rapide qu'à pied) a retenu l'attention des Bolikô de la République Démocratique du Congo. Leur appellation «quatre pieds» s'entend comme «le véhicule qui dédouble vos pieds» et qui vous permet donc d'aller deux fois plus vite. Dans tous les cas, ces appellations ne cherchent pas à rendre compte de la structure schématique (concept archétypique) de la bicyclette. Les différents signifiés attachés aux différents signifiants de ces dénominations correspondent à des points de vue différents sur l'objet, points de vue motivés par et dépendant du passé culturel propre à chaque communauté. Le signifié n'est donc pas l'équivalent du concept, mais seulement un pointeur vers le concept, un point d'ancrage qui permet de saisir globalement le concept, sans avoir à en reconstituer tous les éléments structurels (voir figure ci-après). Nous aimerions pour cette raison l'appeler "percept», comme cela nous a été suggéré à Tunis.

Dans la dichotomie saussurienne signifié/signifiant, le terme "signifié » désigne globalement tout le contenu sémantique attaché à la forme du signe linguistique. Il est encore très couramment utilisé comme synonyme de «concept», voire de "notion». Dans cette interprétation large, il recouvre donc aussi bien notre «percept» que notre «concept». Mais on peut aussi argumenter que le signe linguistique étant une unité minimale de signification, le signifié qui le compose doit être compris comme une valeur significative minimale. Ce qui permet d'utiliser un signe linguistique entier (avec son signifiant et son signifié) pour exprimer de façon motivée une signification élaborée, un concept, qui peut n'avoir rien à voir avec la valeur minimale du signifié. Cette interprétation restreinte du terme «signifié» à laquelle nous adhérons nous permet de dire qu'en substance le «percept» est un «signifié » dans le cadre de l'unité terminologique qu'est le terme ${ }^{6}$. 


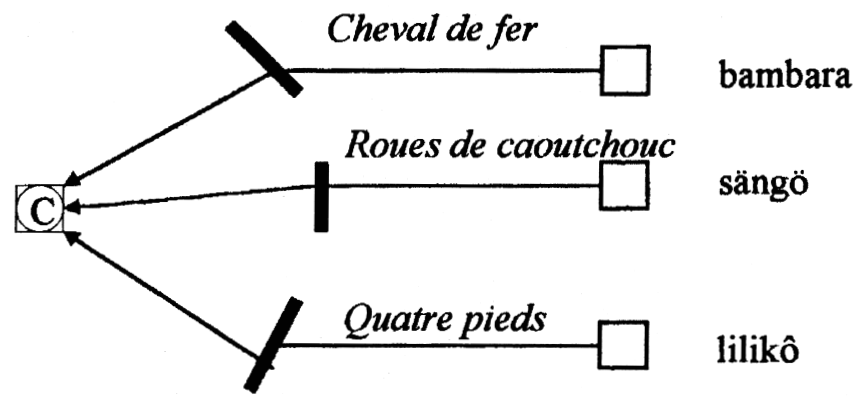

CONCEPT

SIGNIFIÉS

SIGNIFIANTS

\section{CONCLUSION}

Le concept, quelle que soit la définition qu'on lui donne (archétype, pool de traits pertinents, idée essentielle, ensemble de jugements cohérents, etc.), résulte d'une activité mentale d'organisation de l'expérience humaine au sens large, de catégorisation d'objets à des fins d'identification. Le concept permet à l'homme d'élaborer son savoir. Mais l'ensemble des traits pertinents d'un concept ne se retrouve pas forcément dans le mot ou l'expression verbale qui sert à le désigner. La dénomination la plus adéquate, la mieux acceptée, est bien plus souvent celle qui s'intègre le mieux à la langue et à la culture de la communauté des locuteurs. La dénomination apparaît ainsi comme fortement liée à une perception culturelle inscrite essentiellement dans la relation signifiant/signifié ou plus précisément signifiant/percept lorsqu'il s'agit de terminologie et donc de langue de spécialité. Pour aller plus loin, il faudrait creuser davantage la paradoxale relation entre la langue de spécialité et la langue générale. En effet, la langue de spécialité à laquelle appartient totalement le terme est un sousensemble de la langue générale; le terme, on vient de le voir, se doit d'appartenir totalement à la langue dite générale, car c'est la langue tout court. La frontière poreuse entre les sous-ensembles spécialisés de la langue et cet autre sous-ensemble englobant qualifié de langue générale mériterait d'être étudiée plus avant.

\section{NOTES}

1. Cet article a fait l'objet d'une communication aux $\mathrm{V}^{\text {es }}$ Journées scientifiques du réseau Lexicologie, Terminologie, Traduction (LTT) de l'Agence universitaire de la Francophonie à Tunis. La version publiée ici a été très largement revue, corrigée et approfondie.

2. Dans le Dictionnaire de linguistique et des sciences du langage (DLSL) de Dubois et alii (1994), on peut lire à l'entrée concept: «On donne le nom de concept à toute représentation symbolique, de nature verbale, ayant une signification générale qui convient à toute une série d'objets concrets possédant des propriétés communes. » Il est évident que cette définition se veut ramassée et aussi didactique que possible. On trouvera une discussion plus avancée d'une série de définitions du mot concept dans Rey (1979). Nous n'avons évidemment pas la prétention, et encore moins l'intention, d'exposer ici toutes les définitions formulées par les différents courants théoriques à propos du concept. Il nous suffit ici de montrer comment cet axe du concept se distingue et s'articule avec l'axe du signifié (lui-même distinct de l'axe du signifiant).

3. Lorsque nous disons "prioritairement», nous entendons par là que le sens donné est le premier dans le temps depuis l'apparition du mot souris dans la langue française; et lorsque nous disons "prototypiquement», nous faisons allusion au fait que c'est le sens qui vient à l'esprit de la plupart 
des gens quand on leur demande ce que souris veut dire. Il se trouve que des deux points de vue, on obtient le même sens que nous considérerons donc comme sens central par opposition aux autres sens qui, eux, seront à tout le moins non centraux.

4. Information confirmée par Gérard Galtier.

5. Information donnée par Edema Atibakwa.

6. Voir la définition de terme donnée dans l'introduction.

\section{RÉFÉRENCES}

Borsson, Claude et Philippe Thorron (dir.) (1997): Autour de la dénomination, coll. «Travaux du C.R.T.T.», Lyon, Presses universitaires de Lyon.

CABré, Teresa (1997): «Éléments pour une théorie de la terminologie», dans TIA97, II èmes Rencontres terminologies et intelligences artificielles, Université Toulouse-le-Mirail, 24 avril 1997, (sous presse).

Dubois, Jean, Mathée Giacomo, Louis Guespin, Christiane Marcellesi, Jean-Baptiste MarcelLesi, et Jean-Pierre Mevel (1994): Dictionnaire de linguistique et des sciences du langage, Paris, Larousse.

KLeIBER, Georges (1990): La sémantique du prototype: catégories et sens lexical, Paris, PUF.

Duвors, Danièle (dir.) (1991): Sémantique et cognition: catégories, prototype, typicalité, Paris, Éditions du CNRS.

Lakoff, George (1990): Women, Fire, and Dangerous Things: What Categories Reveal About the Mind, Chicago, The Chicago University Press.

Lakoff, George et M. Johnson (1985): Les métaphores dans la vie quotidienne, Paris, Éditions de Minuit.

LANGACKer, Ronald W. (1991) : Concept, Image and Symbol, The Cognitive Basis of Grammar, coll. "Cognitive Linguistics Research", Berlin, Mouton / De Gruyer.

Littré, Émile (1994): Dictionnaire de la langue française, Paris, Éditions de la Fontaine au Roi. Rey, Alain (1979): La terminologie: noms et notions, coll. «Que sais-je?», Paris, PUF. 